\title{
Penile Hair Tourniquet Syndrome Due to a Coil of Hair: First Report in
}

\section{Iran}

\author{
Mohammad Sobhan Sheikh Andalibi, ${ }^{1}$ Anoush Azarfar, ${ }^{2}$ Mohammad Esmaeeli, ${ }^{2}$ Nazanin Sheykh \\ Andalibi, ${ }^{2}$ Anahita Alizadeh, ${ }^{3}$ and Yalda Ravanshad ${ }^{4,}$ \\ ${ }^{1}$ Student Research Committee, Cardiovascular Research Center, School of Medicine, Mashhad University of Medical Sciences, Mashhad, Iran \\ ${ }^{2}$ Departments of Pediatrics, School of Medicine, Mashhad University of Medical Sciences, Mashhad, Iran \\ ${ }^{3}$ Paediatrician, Clinical Toxicology Fellowship, Assistant Professor, Department of Clinical Toxicology, Imam Reza Hospital, School of Medicine, Mashhad University of \\ Medical Sciences, Mashhad, Iran \\ ${ }^{4}$ Clinical Research Unit, Mashhad University of Medical Sciences, Mashhad, Iran \\ "Corresponding author: Yalda Ravanshad, Clinical Research Unit, Mashhad University of Medical Sciences, Mashhad, Iran. Tel: +98-5137269021-Ext 155, E-mail: \\ azarfara@mums.ac.ir
}

Received 2016 December 16; Revised 2017 April 17; Accepted 2017 June 10.

\begin{abstract}
Penile hair tourniquet syndrome (PHTS) is a rare clinical condition characterized by progressive penile strangulation caused by a piece of hair curled around sulcus corona. This clinical condition may result in some clinical and urgent complications including ischemia and gangrene of glans penis, urethra-cutaneous fistulae, and urethral transection. The case report is about a four year and six month old circumcised boy with a hair coil penile strangulation caused by strands of hair being wrapped around the sulcus corona.
\end{abstract}

Keywords: Penile Hair Tourniquet Syndrome, Strangulation, Iran

\section{Introduction}

Tissue strangulation by a thread was first introduced by Guillimeau in 1612 (1) and it was first reported and published in the Lancet in 1832 (2). Penile hair tourniquet syndrome (PHTS) is a rare condition characterized by a progressive hair coil penile strangulation mainly occurring in toddlers (3). This clinical situation is also known as "progressive penile strangulation, hair coil penile strangulation and penile tourniquet syndrome" (4). The penile strangulation represents $25 \%$ of individuals with PHTS and is usually seen in external parts of genitalia (5). This condition results in partial or total obstruction of circulation, ischemia and then, necrosis of the strangulated organ.

Harouchi et al. in 1980 (6) reported the largest series of PHTS and recently some studies reported the number of male cases with different degrees of this syndrome (4). Herein, we aimed to report on the diagnosis and the treatment of a 4 year and 6 month old boy with a swollen penis entrapped with a hair strand which is the first report in Iran.

\section{Case Presentation}

The patient's mother recognized an increased swelling and pain in glans penis and sulcus coronalis for the past 24 hours in his 4 year and 6 month old boy who was admitted to the emergency department of Dr. Sheikh hospital. He had irritability during urination, mild penile swelling, and coronal sulcus erythema for the last two weeks.

His parents did not declare a history of remarkable trauma, foreign body, allergy, and other important disorders or surgery for their son, except nocturnal enuresis and circumcision under local anaesthesia when he was 27 days old. On the examination, the patient was irritable and there was a penile swelling at the glans, and there was an erythematic and tender circumferential line at the coronal sulcus. Fortunately, his penis was not transected at the coronal sulcus, urethra, corpus covernosum and, also, other bundles were normal. Bladder, ureters, and kidneys were normal in ultra-sonographic imaging. In addition, lab findings such as blood electrolytes and serum creatinine and urinalysis were normal and negative.

At first, oral antibiotics were prescribed. Foreign body and child abuse were the initial diagnosis due to a coiled circumferential hair-thread strangulating the glans. The child was taken to the outpatient operation room; examination showed a hair tourniquet lodged in the coronal level without any fistula and penis was uncoiled with a needle (Figure 1). 


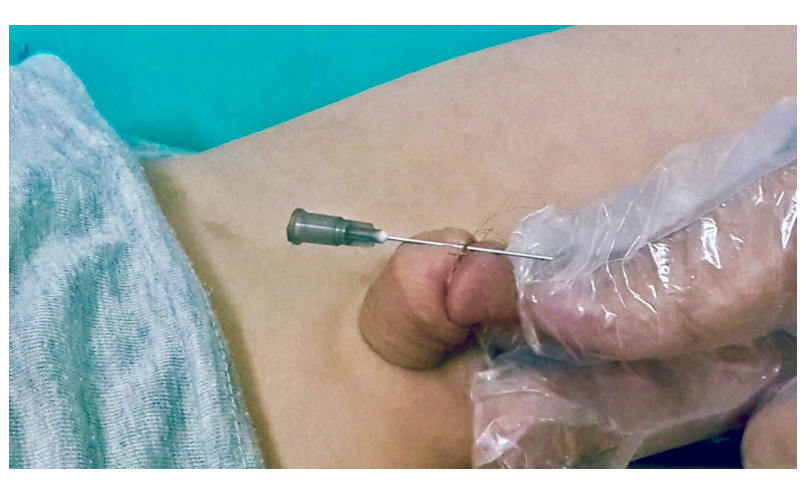

Figure 1. Coiled Hairs in the Coronal Sulcus Caused Penile Strangulation

\section{Discussion}

Penile tourniquet injury is a rare entity caused by coiling of numerous foreign objects including: rubber bands, hair, bottles, lint, thread, and pieces of cloth around the coronal sulcus in circumcised boys (7). There are some studies reporting the complications of this syndrome ranging from a mild penile edema to penile necrosis and amputation (4). Hair penile strangulation is the most common and potentially devastating form of penile tourniquet syndrome (4). Of note, early diagnosis and management improve the prognosis of the patients (4).

According to the literature, hair-thread tourniquet syndrome occurs in boys but some studies have reported this syndrome in girls (8). Circumcision, lack of cleanliness, a moist environment, and childhood are important predisposing factors $(5,7)$. In addition, penile hair strangulation is easier for circumcised patients than around a sulcus with an intact prepuce (9). However, Sallami et al. (10) reported a 37-year-old circumcised man with 10-year post coital pain without any history of trauma or foreign body. He had a history of penile strangulation 34 years ago which was removed at that time. There was not any considerable change in the shape, colour, and sensation of his penis and after surgery his sexual function and voiding was normal.

As Haddad et al. (9) have reported and reviewed the common causes of penile entrapment, there are four major etiologies for penile strangulation including accidental, incidental, intentional, and undetermined. However, it was reported in a 7-year-old uncircumcised boy whose penis was strangulated by the ligation of thread at the base of penis with complete urethral transaction and partial penile amputation (11).

Human hair which is one of the causes of PHTS is extremely thin and therefore easily coiled, especially in the presence of foreign body reaction and swelling. Furthermore, environmental situations such as humidity affect human hair length as it may be expanded and contracted or tightened when being wet and dry, respectively (7). These features of human hair make it an appropriate agent for accidental or intentional constriction (7). Understanding this etiologic mechanism can be helpful by early diagnosing and preventing the progression of severe complications such as penile amputation (4). However, at the early stage, the hair is hidden due to a swollen penile and, therefore, cannot be easily recognized (12).

Badawy and Soliman (4) reported the major complication of PHTS in 25 cases. They observed that all circumcised patients had transection of the urethra at the coronal sulcus. Our case did not have this complication, which may be due to an urgent diagnosis and treatment.

It should be noted that cultural beliefs may play a part in PHTS (10). Some studies reported that parents put a hair around the distal part of their son's penis to improve the sexual function in adulthood (10). In some cases, it occurs accidentally or intentionally by patient's parents, siblings or even by himself (4). For instance, parents have tied up hair around a penis to control enuresis in their child (7). In our case the exact reason why the patient had a tight hair around his sulcus coronalis remained unclear. However, some cultural and criminal issues mentioned above were ruled out. In these situations, clinicians must be alerted about the psychosocial implications of this condition and envisaged about criminal issues such as child abuse.

\subsection{Conclusion}

PHTS should be contemplated as a differential diagnosis of swollen penis or extremities when systemic problems are ruled out. Prompt diagnosis and urgent management can reduce the complications such as loss of appendages and transection of urethra. In early stages and non-deep tourniquets, local care wound and obviation of hair by outpatient surgery would have favourable efficacy. In deeply imbedded constrictors, corporal body or secondary urethral fistula reconstruction is necessary.

\section{Acknowledgments}

We are thankful to the department of pediatric nephrology at Dr. Sheykh hospital, Mashhad University of Medical Sciences (MUMS), as well as research Council of MUMS for their clinical and financial support.

\section{References}

1. Sheinfeld J, Cos L, Erturk E, Cockett A. Penile tourniquet injury due to a coil of hair. J Urol. 1985;133(6):1042-3.

2. The Lancet. . Ligature of the penis. $1832 ; 2(136)$. 
3. Zengin K, Ozdamar MY, Albayrak S, Tanik S, Atar M, Bakirtas H. Hair coil penile tourniquet syndrome in an unusual age. Case Reports Urol. 2015

4. Badawy H, Soliman A, Ouf A, Hammad A, Orabi S, Hanno A. Progressive hair coil penile tourniquet syndrome: multicenter experience with 25 cases. J Pediatr Surg. 2010;45(7):1514-8. doi: 10.1016/j.jpedsurg.2009.11.008. [PubMed: 20638535].

5. Acimi S. Penile strangulation by hair. Pediatr Surg Int. 2014;30(7):72932. doi: 10.1007/s00383-014-3523-9. [PubMed: 24879557].

6. Harouchi A, El-Andaloussi M, Benhayoun N. Les strangulations du gland par cheveu. Int Magh Medical. 1980;2:19-26.

7. Chaware SM, Gajbhiye R, Singh AK. Penile tourniquet injury due to a coil of hair. Indian J Plast Surg. 2006;39(1):70. doi: 10.4103/09700358.26909.

8. Mat Saad AZ, Purcell EM, McCann JJ. Hair-thread tourniquet syn- drome in an infant with bony erosion: a case report, literature review, and meta-analysis. Ann Plast Surg. 2006;57(4):447-52. doi: 10.1097/01.sap.0000222571.98387.71. [PubMed:16998341].

9. Haddad FS. Penile strangulation by human hair. Report of three cases and review of the literature. Urol Int. 1982;37(6):375-88. [PubMed: 7179601].

10. Sallami S, Ben Rhouma S, Cherif K, Noura Y. Hair-thread tourniquet syndrome in an adult penis: case report and review of literature. Urol J. 2013;10(2):915-8. [PubMed: 23801480].

11. Imran D, Ahmed J, Shah T. Delayed presentation of paediatric penile constrictive band injury. British J Plast Surg. 2003;56(3):300-2. doi: 10.1016/s0007-1226(03)00113-9.

12. Jesus LE, Braganca JJ, Rocha JM, Dekermacher S, Anderson KM. Penile hair tourniquet resulting in hypospadias failure. Indian J Urol. 2014;30(3):345-7. doi: 10.4103/0970-1591.128508. [PubMed: 25097325]. 